\title{
Thermally resistant waveguides fabricated in Nd:YAG ceramics by crossing femtosecond damage filaments
}

\author{
A. Benayas, ${ }^{1}$ W. F. Silva, ${ }^{2}$ C. Jacinto, ${ }^{2}$ E. Cantelar, ${ }^{1}$ J. Lamela, ${ }^{1}$ F. Jaque, ${ }^{1}$ J. R. Vázquez de Aldana, ${ }^{3}$ \\ G. A. Torchia, ${ }^{3}$ L. Roso, ${ }^{3}$ A. A. Kaminskii, ${ }^{4}$ and D. Jaque ${ }^{1}$ \\ ${ }^{1}$ Departamento de Física de Materiales, Facultad de Ciencias, Universidad Autónoma de Madrid, \\ Madrid 28049, Spain \\ ${ }^{2}$ Grupo de Fotônica e Fluidos Complexos, Instituto de Física, Univiversidade Federal de Alagoas, 57072-970, \\ Maceio, Alagoas, Brazil \\ ${ }^{3}$ Grupo de Optica, Facultad de Ciencias, Universidad de Salamanca, Salamanca 37008, Spain \\ ${ }^{4}$ Institute of Crystallography, Russian Academy of Sciences, Moscow 119333, Russia
}

Received August 25, 2009; revised November 16, 2009; accepted December 1, 2009; posted December 22, 2009 (Doc. ID 116238); published January 26, 2010

We report on femtosecond laser writing of channel waveguides in $\mathrm{Nd}^{3+}$ ion doped YAG ceramics by multiple inscriptions of damage filaments. Waveguiding between filaments was found to resist annealing temperatures as high as $1500^{\circ} \mathrm{C}$. Microluminescence imaging experiments have been carried out to elucidate the potential application of the obtained waveguides as integrated laser sources as well as to elucidate the waveguiding mechanisms. (C) 2010 Optical Society of America

OCIS codes: $230.7380,180.2520,160.3380$.

Neodymium-doped transparent YAG ceramics (hereafter Nd:cYAG) is nowadays one of the most promising laser materials with outstanding properties [1-3]. The future incorporation of Nd:cYAG in compact devices requires the fabrication of channel waveguides in which the $2 \mathrm{D}$ light confinement would lead to a much higher optical intensities. Among the different methods already used for the fabrication of channel waveguides in the Nd:cYAG system [4], direct laser writing with femtosecond pulses is of special relevance because of its reduced processing times, its simplicity, and its true 3D versatility [5]. In the case of a Nd:cYAG system, waveguides based on the so-called double-line approach have shown excellent optical and superior laser properties [6,7]. The main drawback of these waveguides is the instability of the strain-field-induced refractive index increment, i.e., of the waveguide, against thermally induced lattice relaxation [6]. This is a negative aspect in laser waveguides, where relevant pump induced thermal loadings are expected to occur [8].

In this Letter we report on the fabrication of thermally resistant Nd:cYAG waveguides by an approach consisting of the consecutive inscription of two orthogonal pairs of femtosecond filaments. The ability of the obtained structure for light confinement and its thermal stability has been investigated. The potential application of the obtained waveguides as laser sources has been explored by luminescence imaging experiments.

The Nd:cYAG sample used in this work was provided by Baikowskii Ltd. (Japan) and was a $1 \times 1$ $\times 1 \mathrm{~cm}^{3}$ prism with a nominal $\mathrm{Nd}^{3+}$ concentration of 2 at. \%. Damage filaments were inscribed by using an amplified Ti:sapphire laser system providing $120 \mathrm{fs}$ pulses linearly polarized at $796 \mathrm{~nm}$ and $1 \mathrm{kHz}$ of repetition rate. The laser beam was focused $250 \mu \mathrm{m}$ below the surface with a $10 \times$ microscope objective
$(\mathrm{NA}=0.3)$. The on-target pulse energy was set to $3 \mu \mathrm{J}$, leading to $\sim 40-\mu \mathrm{m}$-long filaments. In a first step, two filaments were written separated $30 \mu \mathrm{m}$ by translating the sample in the direction perpendicular to the laser polarization and the pulse propagation, with a speed of $50 \mu \mathrm{m} / \mathrm{s}$. Then the sample was turned $90^{\circ}$ and a second pair of filaments, overlapping to the first one was inscribed. The end-face of the obtained quadruple-line structure is shown in Fig. 1(a). The ability of the obtained structure as an optical waveguide was investigated by both endcoupling experiments using a $\mathrm{He}-\mathrm{Ne}$ laser [Fig. 1(b)] as well as by transmission scanning near-field microscopy (SNOM) working at $532 \mathrm{~nm}$ [Fig. 1(c)]. In both cases a strong evidence of waveguiding between the two orthogonal pairs of filaments (indicated by the dashed white lines) was observed. The waveguide's mode seems to be determined and controlled by the position of the filaments. To provide experimental evidence of such control, we fabricated a second waveguide with a reduced filament separation of $15 \mu \mathrm{m}$. The obtained waveguide's mode, which extension is fully given by the separation between filaments, is shown in Fig. 1(d). This control over waveguide's mode is an advantage over double-line waveguides, in which the waveguide's mode is determined not only by the position of the filaments but also by the complex strain field created around them [6]. It should be noted that any polarization was confined by the waveguide. The waveguide's propagation loss was estimated, by measuring the scattering intensity versus propagation length, to be no larger than $2 \mathrm{~dB} / \mathrm{cm}$.

To elucidate which way the multifilament inscription has modified the luminescence properties of neodymium ions, confocal microluminescence experiments were performed. The same experimental setup as that of [6] was used. Figure 2(a) includes the 

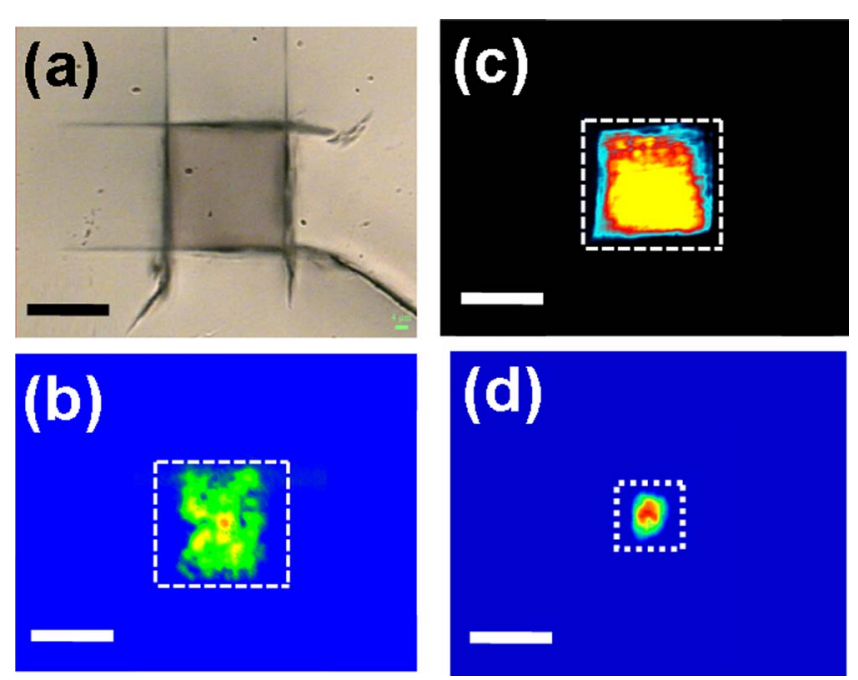

Fig. 1. (Color online) (a) Optical transmission micrograph of a Nd:cYAG multifilament waveguide with a filamentfilament distance of $30 \mu \mathrm{m}$. (b) Near-field intensity distribution of the fundamental mode at $632.8 \mathrm{~nm}$ corresponding to the structure shown in (a). (c) SNOM transmission image of the multifilament waveguide shown in (a). (d) Nearfield intensity distribution of the fundamental mode at $632.8 \mathrm{~nm}$ corresponding to a waveguide with a filamentfilament distance of $15 \mu \mathrm{m}$. In all the cases the white dashed lines indicate the position of the filaments. Scale bar is, in all the cases, $20 \mu \mathrm{m}$.

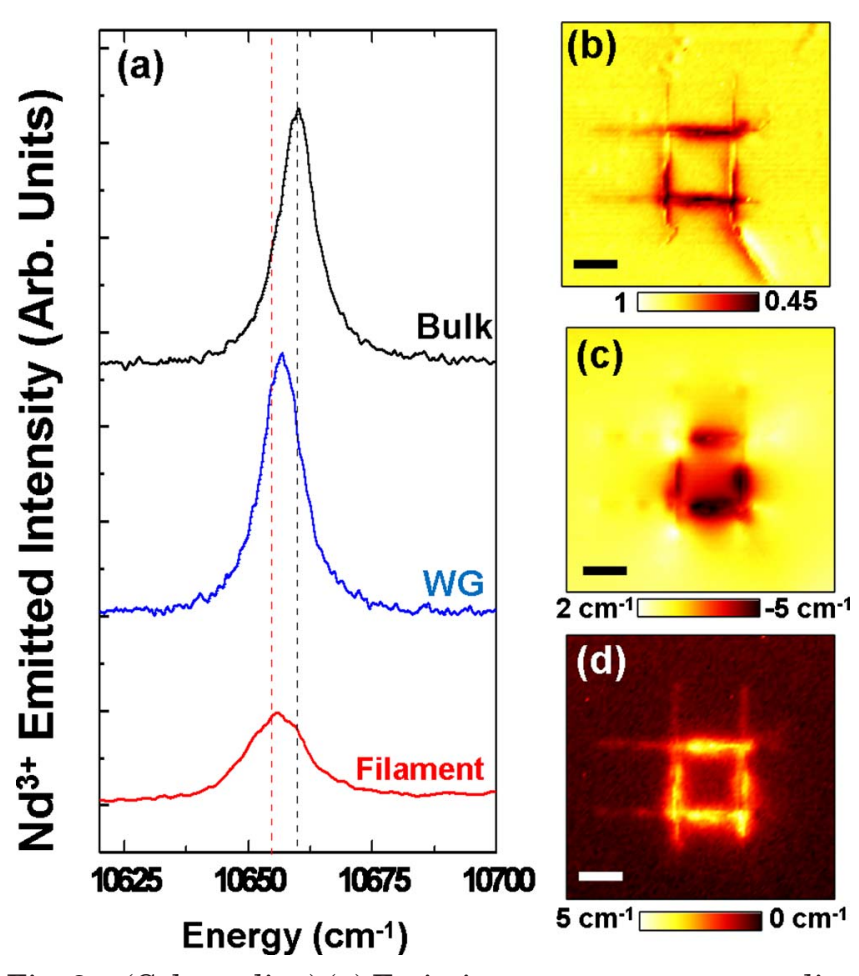

Fig. 2. (Color online) (a) Emission spectrum corresponding to the $\mathrm{Nd}^{3+4} F_{3 / 2}\left(R_{1}\right) \rightarrow{ }^{4} I_{9 / 2}\left(Z_{5}\right)$ laser transition as obtained from a unprocessed area, from the waveguide and from a filament. (b), (c), and (d) are the spatial dependences of the luminescence intensity, induced spectral shift, and induced spectral broadening of the ${ }^{4} F_{3 / 2}\left(R_{1}\right) \rightarrow{ }^{4} I_{9 / 2}\left(Z_{5}\right) \mathrm{Nd}^{3+}$ emission line in the as-fabricated waveguide, respectively. Scale bar is, in all the cases, $20 \mu \mathrm{m}$. microluminescence spectra corresponding to the ${ }^{4} F_{3 / 2}\left(R_{1}\right) \rightarrow{ }^{4} I_{9 / 2}\left(Z_{5}\right)$ laser transition of neodymium ions [9], as obtained from an unprocessed area (bulk), from one filament and from the waveguide (area delimited by the four filaments). A first inspection, Fig. 2(a) evidences a clear redshift of this $\mathrm{Nd}^{3+}$ emission line at filament's location being this accompanied by a relevant broadening and intensity reduction. To unequivocally correlate these changes with the location and extension of the filaments, the spatial distribution of the intensity, spectral shift, and bandwidth of this $\mathrm{Nd}^{3+}$ emission line have been measured [Figs. 2(b)-2(d), respectively]. Since luminescence reduction, spectral redshifting and line broadening are unequivocally related to lattice damage [10], lattice compression [11], and local disordering, respectively, the luminescence maps of Fig. 2 indicate that a relevant damage and disordering of the Nd:cYAG network has been locally produced at filaments. The absence of any luminescence quenching at waveguide's location indicates that the obtained structure is a promising candidate for integrated laser action. Whereas the induced damage and disordering is well localized at filaments, the induced lattice compression (denoted by the luminescence redshift) spreads out, extending over the waveguide's area. Taking into account the pressure coefficient of the ${ }^{4} F_{3 / 2}\left(R_{1}\right)$ $\rightarrow{ }^{4} I_{9 / 2}\left(Z_{5}\right)$ luminescence line $\left(\sim 0.75 \mathrm{~cm}^{-1} / \mathrm{Kbar},[10]\right)$ we can estimate that a residual compressive stress of 3.75 and $2.75 \mathrm{Kbar}$ has been induced at the filaments and at the waveguide, respectively. The refractive index map that can be inferred from the luminescence images of Fig. 2, according to previous works [5], is constituted by a refractive index reduction at the damaged filaments (of the order of $\Delta n_{\text {damage }} \sim-10^{-2}$ ) and by a pressure induced refractive index increment at waveguide's volume (of the order of $\Delta n_{\text {pressure }} \sim+10^{-3}$ ).

To check the thermal resistance of the waveguide, it was annealed for $4 \mathrm{~h}$ at different temperatures ranging from $200^{\circ} \mathrm{C}$ up to $1500^{\circ} \mathrm{C}$, i.e., up to $0.75 T_{m}$, $T_{m}$ being the YAG's melting point $\left(\sim 2000^{\circ} \mathrm{C}\right)$. The variation of the aspect ratio of the waveguide's mode (defined as the ratio between vertical and horizontal mode extensions) for the different annealing temperatures is shown in Fig. 3(a). We have found that the shape of waveguide's mode is thermally stable. This can be clearly observed when comparing the $632 \mathrm{~nm}$ waveguide's modes obtained in the as-fabricated structure and after the $1500^{\circ} \mathrm{C}$ annealing [bottom insets in Fig. 3(a)]. This is an advantage over the previously fabricated doublelinewaveguides in which the aspect ratio of the waveguide's mode was already strongly affected for relatively low annealing temperatures of $1000^{\circ} \mathrm{C}$ due to the thermal erasing of the strain field [see Fig. 3(a)] [6]. To make this fact more evident we have included in Fig. 3(a) the waveguide's modes obtained from a double-line waveguide before and after $1500^{\circ} \mathrm{C}$ annealing. The luminescence images obtained from the quadruple-linestructure after $1500^{\circ} \mathrm{C}$ annealing are shown in Figs. $3(\mathrm{~b})-3(\mathrm{~d})$. It is 


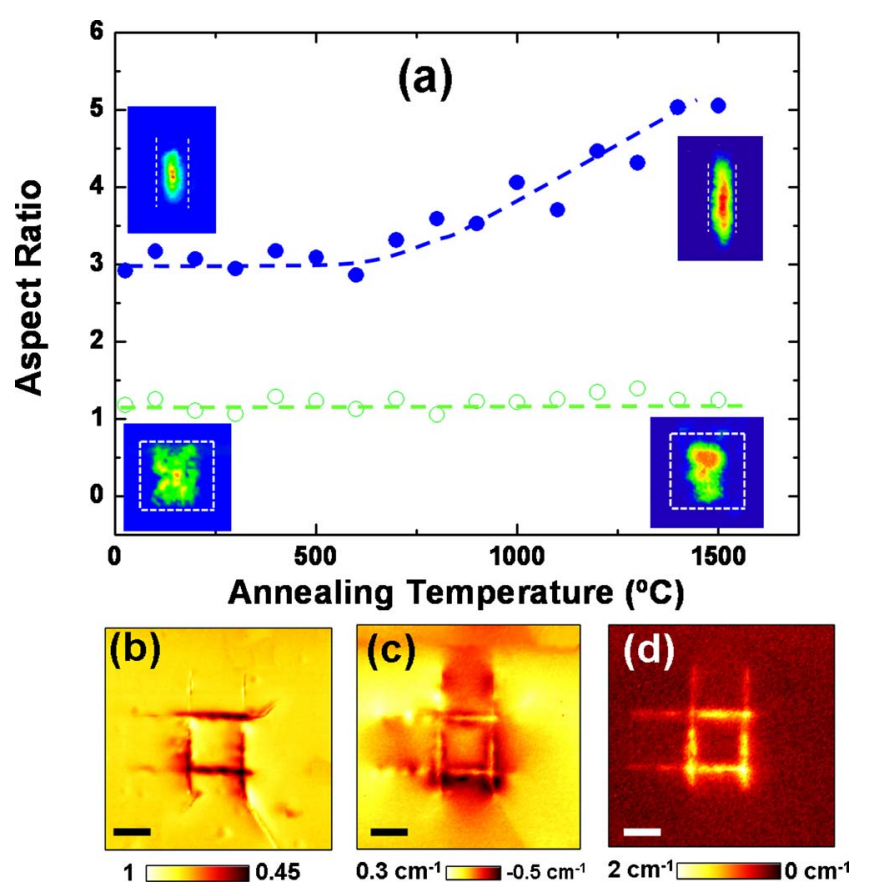

Fig. 3. (Color online) (a) Variation of the aspect ratio of the waveguide's mode (defined as the ratio between vertical and horizontal mode extensions) for the different annealing temperatures as obtained for double-line and quadrupleline waveguides (solid and open circles, respectively). The insets are the waveguide's modes for each case as obtained from the as-fabricated structures and after $1500^{\circ} \mathrm{C}$ annealing. Dashed lines indicate the location of filaments. (b), (c), and (d) are the spatial dependence of the luminescence intensity, induced spectral shift, and induced spectral broadening of the ${ }^{4} F_{3 / 2}\left(R_{1}\right) \rightarrow{ }^{4} I_{9 / 2}\left(Z_{5}\right) \mathrm{Nd}^{3+}$ emission line as obtained from a quadruple-line waveguide after thermal annealing at $1500^{\circ} \mathrm{C}$ for $4 \mathrm{~h}$, respectively. The filamentfilament separation is $30 \mu \mathrm{m}$, and the scale bar is, in all the cases, $20 \mu \mathrm{m}$.

clear that the local damage created at filaments (causing the luminescence reduction) is not thermally reversible [see Fig. 3(b)]. On the other hand, the induced spectral shift [Fig. 3(c)] has been drastically modified in respect to that obtained from the asfabricated structure [see Fig. 2(c)]. According to previous discussion, we can conclude that the thermal annealing has reduced by 1 order of magnitude (from 3.75 down to $0.3 \mathrm{Kbar}$ ), the maximum induced compressive stress in the close surroundings of the filaments, and has almost completely removed the induced compression at waveguide's location. Finally, the comparison between the luminescence images of Figs. 2(d) and 3(d) reveals a noticeable reordering of the Nd:cYAG network as a consequence of the ther- mal annealing. Thus, it is clear that the $1500^{\circ} \mathrm{C}$ thermal annealing does not remove the damage-induced refractive index reduction produced at filaments.

In summary, we have demonstrated an approach for the fabrication of channel waveguides in Nd:YAG ceramics consisting of multiple inscription of damage filaments by femtosecond pulses. The reported structures have shown an excellent optical confinement that is thermally stable up to temperatures as high as $1500^{\circ} \mathrm{C}$. We have provided experimental evidence that the luminescence efficiency of neodymium ions at the waveguide's volume is not affected by the multifilament inscription. In addition, the waveguide's luminescence images have provided information about the different mechanism modifying the refractive index. In as-fabricated structures the refractive index was modified both at the filaments (due to local damage) and in the region limited by them (due to local compression), whereas after $1500^{\circ} \mathrm{C}$ thermal annealing the strain field is almost completely removed in such a way that waveguiding is provided by the reduced index walls $\left(\Delta n_{\text {damage }} \sim-10^{-2}\right)$ constituted by the filaments.

This work has been supported by the Spanish Ministerio de Ciencia y Tecnología (MAT2007-64686 and CSD2007-00013), the Consejería de Educación de la Comunidad de Madrid, Universidad Autónoma de Madrid (UAM-CAM) (CCG08-UAM/MAT-4434), CEAL-Banco Santander, and CAPES and CNPq.

\section{References}

1. A. Ikesue and Y. L. Aung, J. Am. Ceram. Soc. 89, 1936 (2006).

2. A. A. Kaminskii, Laser Photonics Rev. 1, 87 (2007).

3. J. Lu, M. Prabhu, J. Song, C. Li, J. Xu, K. Ueda, A. A. Kaminskii, H. Yagi, and T. Yanagitani, Appl. Phys. B 71, 469 (2000).

4. F. Chen, Y. Tang, and D. Jaque, Opt. Lett. 34, 28 (2009).

5. S. Nolte, M. Will, J. Burghoff, and A. Tuennermann, Appl. Phys. A 77, 109 (2003).

6. A. Ródenas, G. A. Torchia, G. Lifante, E. Cantelar, J. Lamela, F. Jaque, L. Roso, and D. Jaque, Appl. Phys. B 95, 85 (2009).

7. G. A. Torchia, A. Rodenas, A. Benayas, E. Cantelar, L. Roso, and D. Jaque, Appl. Phys. Lett. 92, 111103 (2008).

8. N. Pavel, V. Lupei, J. Saikawa, T. Taira, and H. Kan, Appl. Phys. B 82, 599 (2006).

9. A. A. Kaminskii, Laser Crystals (Springer, 1981).

10. B. Henderson and G. F. Imbusch, Optical Spectroscopy of Inorganic Solids (Oxford Science, 1989).

11. S. Kobyakov, A. Kamińska, A. Suchocki, D. Galanciak, and M. Malinowski, Appl. Phys. Lett. 88, 234102 (2006). 